\title{
Solubilized Cartilage ECM Facilitates the Recruitment and Chondrogenesis of Endogenous BMSCs in Collagen Scaffolds for Enhancing Microfracture Treatment
}

Yan Lu ${ }^{l}$, Yuxiang Wang ${ }^{l}$, Hanjie Zhang ${ }^{l}$, Zizhao Tang ${ }^{1}$, Xiaolin Cui ${ }^{2}$, Xing Li $i^{1}$, Jie Liang ${ }^{l}$, Qiguang Wang ${ }^{1 *}$, Yujiang Fan ${ }^{1 *}$ and Xingdong Zhang ${ }^{1}$

1. National Engineering Research Center for Biomaterials, Sichuan University, 29 Wangjiang Road, Chengdu, Sichuan, 610065, China

2. Department of Orthopaedic Surgery, University of Otago, Christchurch, 8011, New Zealand

Keywords: Cartilage ECM, Decellularization, Collagen scaffold, Chondrogenesis, Microfracture, Cartilage repair

\section{Corresponding Author}

*Email: wqgwang@126.com or fan_yujiang@scu.edu.cn 
Table S1. Sequence of related gene primers.

\begin{tabular}{ccc} 
Gene & Forward primer & Reverse primer \\
\hline GAPDH & TCGGAGTGAACGGATTTGGC & TTCCCGTTCTCAGCCTTGAC \\
Col I & GTCGATGGCTGCACGAAAAA & GGGCCAACGTCCACATAGAA \\
Col II & TGATAAGGATGTGTGGAAGCCG & CAGGCAGTCCTTGGTGTCTTC \\
Col X & TCCCAGA ACCCAGAATCCATC & GGTTGTGGGCCTTTTATGCC \\
Sox 9 & TCTGGAGACTGCTGAACGAG & CTGCCCATTCTTCACCGACTT \\
AGG & GGCCACTGTTACCGTCACTT & GTCCTGAGCGTTGTTGTTGAC \\
\hline
\end{tabular}

Table S2. International Cartilage Repair Society (ICRS) cartilage repair assessment tool.

\begin{tabular}{|c|c|c|}
\hline & Criteria & Points \\
\hline \multirow[t]{5}{*}{ Degree of defect repair } & Level with surrounding cartilage & 4 \\
\hline & $75 \%$ repairof defect depth & 3 \\
\hline & $50 \%$ repair of defect depth & 2 \\
\hline & $25 \%$ repair of defect depth & 1 \\
\hline & $0 \%$ repair of defect depth & 0 \\
\hline \multirow[t]{8}{*}{ Integration to border zone } & Complete integration with surounding cartilage & 4 \\
\hline & Demarcating border $<1 \mathrm{~mm}$ & 3 \\
\hline & $3 / 4$ of graft integrated, $1 / 4$ with a notable border & 2 \\
\hline & $>1 \mathrm{~mm}$ width & \\
\hline & $1 / 2$ of graft integrated with surrounding & 1 \\
\hline & cartilage, $1 / 2$ with a notable border $>1 \mathrm{~mm}$ & \\
\hline & From no contact to $1 / 4$ of graft integrated with & 0 \\
\hline & surrounding cartilage & \\
\hline \multirow[t]{5}{*}{ Macroscopic appearance } & Intact smooth surface & 4 \\
\hline & Fibrillated surface & 3 \\
\hline & Small,scattered fissure or cracks & 2 \\
\hline & Several, smallor few but large fissures & 1 \\
\hline & Total degeneration of grafted area & 0 \\
\hline \multirow[t]{4}{*}{ Overall score } & Grade I normal & 12 \\
\hline & Grade $\mathbb{I}$ nearly normal & $11-8$ \\
\hline & Grade III abnormal & $7-4$ \\
\hline & Grade IV severely abnormal & $3-1$ \\
\hline
\end{tabular}

